\title{
A Porous Multilayer Dye-Based Photoelectrochemical Cell That Unexpectedly Runs in Reverse
}

\author{
Kathryn E. Splan, Aaron M. Massari, and Joseph T. Hupp* \\ Department of Chemistry, 2145 Sheridan Road, Northwestern University, Evanston, Illinois 60208
}

Received: October 24, 2003

Porous dye multilayers based on cavity-containing porphyrinic molecular squares are capable of sensitizing flat indium - tin oxide (ITO) electrodes to photocurrent production with visible light. In aqueous $\mathrm{I}_{3}^{-} / \mathrm{I}^{-}$solutions the sensitized electrodes unexpectedly produce cathodic photocurrents, the opposite of the anodic photocurrents generally observed with conventional dye-senstized solar cells (DSSCs). In constrast to DSSCs, which work by electron injection from a dye excited state into the photoelectrode's conduction band, the mechanism of photocurrent generation is $\mathrm{I}_{3}{ }^{-}$quenching of the porphyrin square excited state to produce an oxidized dye. Quenching is possible, despite the short excited state lifetime, because of ground state donor (chromophore)/ acceptor (quencher) complex formation. Following quenching, ground state redox hopping through the multilayer structure delivers the oxidizing equivalent to the ITO electrode. One consequence is the photocurrent production can be systematically increased by adding dye layers and collecting more light (behavior not usually seen with DSSCs). Another is that only small photovoltages can be produced. A third is that the photocurrent production scheme is inoperable when ITO is replaced by titanium dioxide, because the electrons produced are energetically incapable of reaching the $\mathrm{TiO}_{2}$ conduction band. Perhaps most importantly, to the extent that the first part of the scheme (molecular quenching) occurs in conventional cells, the photocurrent efficiencies of these cells will be diminished.

\section{Introduction}

One of the most promising photochemical approaches to solar energy conversion is dye sensitization of wide band gap semiconductors. ${ }^{1-3}$ The best approaches to date are based on high-area $\mathrm{TiO}_{2}$ electrodes featuring a ruthenium polypyridyl complex as the sensitizer, and the $\mathrm{I}^{-} / \mathrm{I}_{3}{ }^{-}$couple as a dye regenerator and redox shuttle between the photoelectrode and a dark electrode. ${ }^{4-8}$ Though understanding of the fundamental aspects of the operation of high-area dye-sensitized solar cells has advanced significantly over the past 12 years, the cells themselves have proven remarkably resistant to efficiency improvement; the version of the Grätzel cell described in 1991 operated with $7.9 \%$ overall efficiency ${ }^{9}$ - only marginally poorer than contemporary versions. ${ }^{4,5}$ These versions operate with close to $100 \%$ quantum efficiency; i.e., nearly every photon absorbed yields an electron in the external circuit. The cells also feature an almost optimal match with the solar spectrum, capturing most of the photons available between $\sim 400$ and $775 \mathrm{~nm} .^{5}$ They suffer, however, from low photovoltages - roughly $40-50 \%$ of what is theoretically possible. The photovoltage losses appear to be concentrated in two areas. First, to regenerate the lightabsorbing form of the dye at an acceptable rate, the iodide/ triiodide couple requires a substantial free-energy driving force. ${ }^{10}$ Attempts to replace the couple with kinetically more facile redox couples having more optimal potentials have failed because the oxidized halves of the alternative couples evidently lack the unique ability of the triiodide species to resist recombination with the injected electron. ${ }^{10,11}$

The second source of photovoltage loss appears to be surface states. Injected electrons are very rapidly thermalized, reaching the bottom of the conduction band and then occupying defect

* To whom correspondence should be addressed. E-mail: jthupp@ chem.nwu.edu. Phone: 847-491-3504. Fax: 847-491-7713. states below the conduction band. ${ }^{12,13}$ Rather than delivering electrons at energies corresponding to the conduction band edge, defect-containing photoelectrodes deliver electrons at the quasiFermi level. The quasi-Fermi level is the energy at which a surface state is equally likely to be filled or empty. Increasing the rate of electron injection at steady state (for example, by increasing the light intensity) will raise the quasi-Fermi level and increase the photovoltage. Equivalently, decreasing the number of surface states, while leaving the injection rate unchanged, will raise the quasi-Fermi level and increase the photovoltage.

Current nanocrytalline photoanode fabrication schemes yield high porosity, high surface area (ca. 1,000 $\times$ geometric area), titanium dioxide electrodes having nearly ideal properties for dye-based light collection. ${ }^{14}$ Precisely the properties that make the electrodes so useful for light harvesting, however, leave the electrodes with an extraordinary number of trap sites. In principle, high area, trap laden, nanocrystalline electrodes featuring a single layer of adsorbed dye could be replaced with thin, low area, low trap density (polycrystalline or single crystal) electrodes featuring multiple layers of dye. With significantly fewer traps present, traps at a fully sensitized thin electrode would be saturated and electrons could be collected at energies corresponding to the conduction band edge, thus increasing the photovoltage.

Multilayer sensitization has proven surprisingly ineffective, however, with several multilayers of dye performing no better in terms of photocurrent output than a monolayer. ${ }^{15,16}$ The primary problem with multilayer sensitization appears, in most cases, not to be inefficient layer-to-layer energy transfer, but recombination of the injected electron with the oxidized dye. Recombination obviously has disastrous consequences in terms of quantum efficiency and photocurrent production. Permanent charge separation requires charge compensation and ultimately 
reduction of the oxidized dye by a shuttle species such as iodide ion. The challenge is to find a structural motif that permits ions to be delivered rapidly to the oxidized dye. The Grätzel cell accomplishes this by infusing a nanoporous electrode assembly with an electrolyte solution. The analogous strategy for a flat electrode geometry is to infuse nanoporous dye multilayers with an electrolyte solution.

We recently reported on the fabrication of ultrathin porphyrin films based on the diphosponic acid porphyrin $1 .{ }^{17}$ The assembly

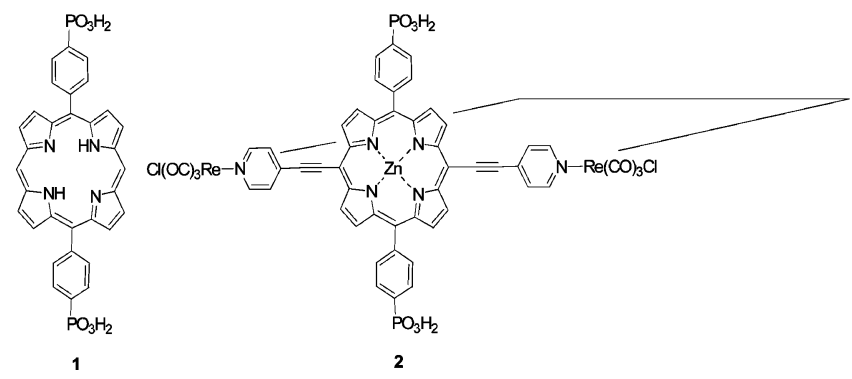

scheme, which utilizes well-established zirconium phosphonate chemistry, ${ }^{18-20}$ yields highly oriented films (normal to the surface) of well-defined thickness. Extension of the fabrication chemistry to the analogous porphyrin square compound $\mathbf{2}$ yields films that are similarly well defined. We reasoned that the porosity and molecular permeability of the square films would render them suitable for multilayer sensitization of low area photoelectrodes. As part of such studies we unexpectedly encountered conditions where photocurrents are reversed. In other words, the illuminated electrode behaves as a photocathode rather than photoanode. We report here on the mechanistic basis for photocurrent reversal and consider the implications of the observation for the performance of dye-sensitized solar cells (DSSCs) displaying conventional photocurrent responses.

\section{Experimental Section}

Materials. Porphyrin compound $\mathbf{1}$ was prepared as described previously. ${ }^{17}$ Samples of molecular square 2 were provided by Dr. Rich Gurney. Sodium iodide (Fisher Scientific) and iodine (Mallinckrodt) were used as received. Water was purified using a Millipore filtration and ion-exchange system and had a resistivity of $18.2 \mathrm{M} \Omega$. Conductive glass (indium-tin oxide (ITO) coated glass) was purchased from Delta Technologies, Ltd.

Thin Film Preparation. Thin films of $\mathbf{2}$ were prepared and characterized analogously to the published procedure for $\mathbf{1}^{17}$ Briefly, phosphorylated ITO (or $\mathrm{TiO}_{2}$ ) electrodes were immersed overnight in $25 \mathrm{mM} \mathrm{ZrOCl}_{2}$ (aq). The platforms were then immersed in a $0.1 \mathrm{mM}$ dimethyl sulfoxide (DMSO) solution of 2 for $4 \mathrm{~h}$. Following each exposure to $\mathrm{Zr}^{4+}$ or porphyrin, the substrates were rinsed in $\mathrm{H}_{2} \mathrm{O}$ or DMSO, respectively. Additional layers were assembled through successive treatment with $25 \mathrm{mM} \mathrm{ZrOCl}_{2}(15 \mathrm{~min})$ and $0.1 \mathrm{mM} \mathrm{2}$, either by manual means or by using a programmed robotic arm. $\mathrm{TiO}_{2}$ precursor sols were synthesized using $\mathrm{Ti}\left(\mathrm{O}-{ }^{n} \mathrm{Bu}\right)_{4}$ in the presence of acetic acid and acetylacetone according to a literature procedure. ${ }^{21}$ Solgel $\mathrm{TiO}_{2}$ electrodes were then prepared by spin-casting the precursor sol on ITO substrates and subsequently annealing at $450{ }^{\circ} \mathrm{C}$. To prepare mixed $\mathrm{ITO} / \mathrm{TiO}_{2}$ electrodes, a portion of the ITO substrate was masked with adhesive tape to prevent deposition of $\mathrm{TiO}_{2}$.

Spectral and Photoelectrochemical Measurements. Electronic absorption spectra were obtained on a Varian Cary 5000 UV-vis-NIR spectrophotometer. Steady state fluorescence

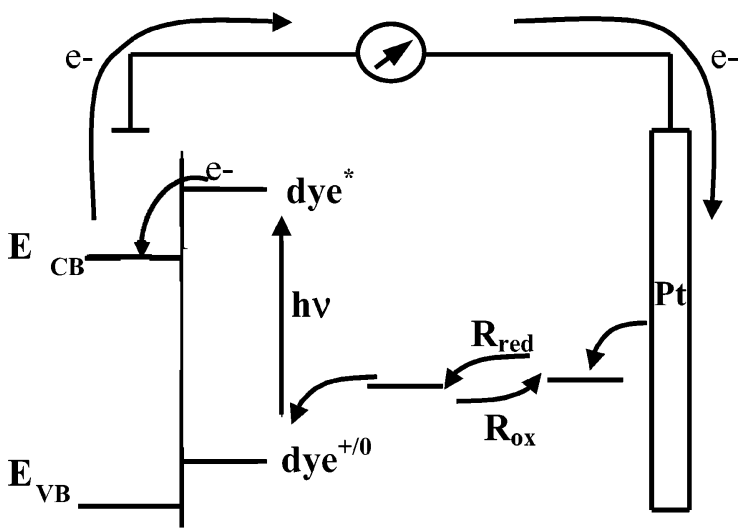

Figure 1. A schematic representation of the operational mechanism of a dye-sensitized solar cell (DSSC).

measurements were performed on a Jobin Yvon-SPEX Fluorolog-3 spectrofluorometer. All photoelectrochemical experiments were conducted in aqueous electrolyte containing $0.5 \mathrm{M}$ $\mathrm{NaI}$ and $0.005 \mathrm{M} \mathrm{I}_{2}$ unless otherwise stated. Photoexcitation of porphyrin electrodes was accomplished using the $450 \mathrm{~W}$ Xe arc lamp and excitation monochromator of a Jobin Yvon-SPEX Fluorolog-3 spectrofluorometer. Photocurrents were measured from the output of a $\mathrm{CH}$ Instruments potentiostat using a two electrode configuration with the photoelectrode held at $0 \mathrm{~V}$ versus the Pt counter electrode. Because films can grow on both sides of the ITO/glass substrates, absorbance values were halved when the fraction of light absorbed by the sensitized electrode was calculated (appropriate for small absorbances).

\section{Results}

Figure 1 illustrates the conventional light-to-electrical energy conversion scheme for DSSCs. As we will report in detail elsewhere, cells based on sensitization of $\mathrm{TiO}_{2}$ with multilayers of $\mathbf{2}$ in acetonitrile as solvent function according to this scheme. As part of these studies, however, we also had occasion to examine the photoresponses of multilayer-2/ITO cells in water as solvent. ITO electrodes are sufficiently heavily doped that dye excited state/semiconductor band-edge energy matching is unnecessary (i.e., a continuum of empty electronic states exists, starting immediately above the Fermi level). It follows that injected electrons will rapidly relax to the energy of the Fermi level, precluding significant photovoltage generation and energy storage. Nevertheless, photoelectrodes and photocells of this kind are of interest because of the conductivity of the underlying platforms even under dark conditions, and the ability of the platforms, therefore, to report electrochemically on film permeability with respect to candidate redox shuttle species. ${ }^{17}$

Shown in Figure 2 is the short-circuit photoresponse of a cell featuring two layers of dye. A small cathodic photocurrent flows in the presence of aqueous $0.5 \mathrm{M} \mathrm{NaI}$, but a much larger current appears when $0.005 \mathrm{M} \mathrm{I}_{2}$ (primarily in the form of $\mathrm{I}_{3}{ }^{-}$) is also present. Shown in the inset is the $I_{2}$ concentration dependence of the photoresponse. Notably, the response plateaus at several millimolar. Voltammetry experiments (not shown) establish that both $\mathrm{I}^{-}$and $\mathrm{I}_{3}{ }^{-}$are able to permeate the dye coating and engage in electron transfer with the underlying ITO electrode.

Figure 3, a photocurrent action spectrum, establishes that the light absorber is the porphyrin square coating. Figure 4 shows plots of photocurrent versus fraction of light absorbed, where light absorption is systematically increased as more layers of $\mathbf{2}$ are added. Remarkably, even with 15 layers of dye, the photocurrent magnitude continues to scale with the fraction of incident light absorbed. 


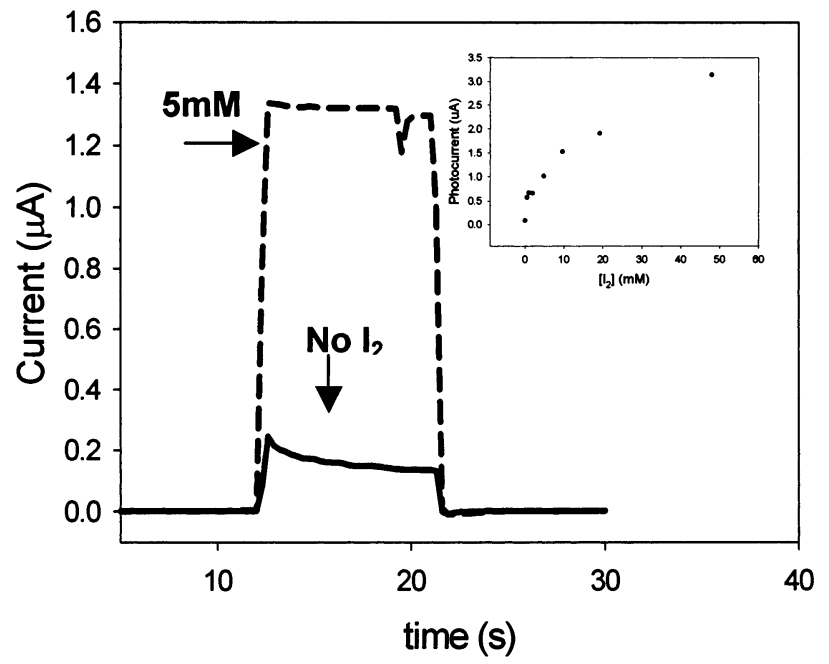

Figure 2. Short-circuit photoresponse for a two-layer film of 2. Inset: Photocurrent generation (450 $\mathrm{nm}$ excitation) as a function of $\mathrm{I}_{2}$ concentration.

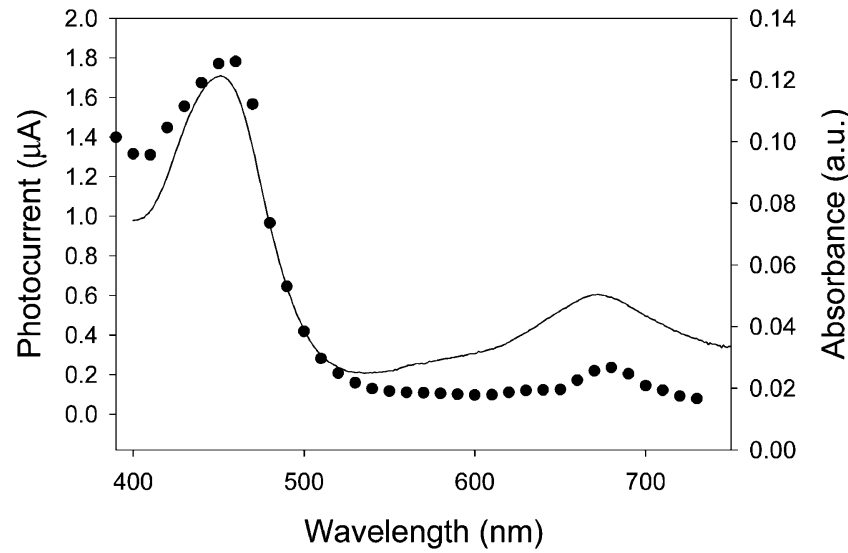

Figure 3. Photocurrent action spectrum $(\cdots)$ and absorption spectrum $(-)$ of a two-layer film of 2.

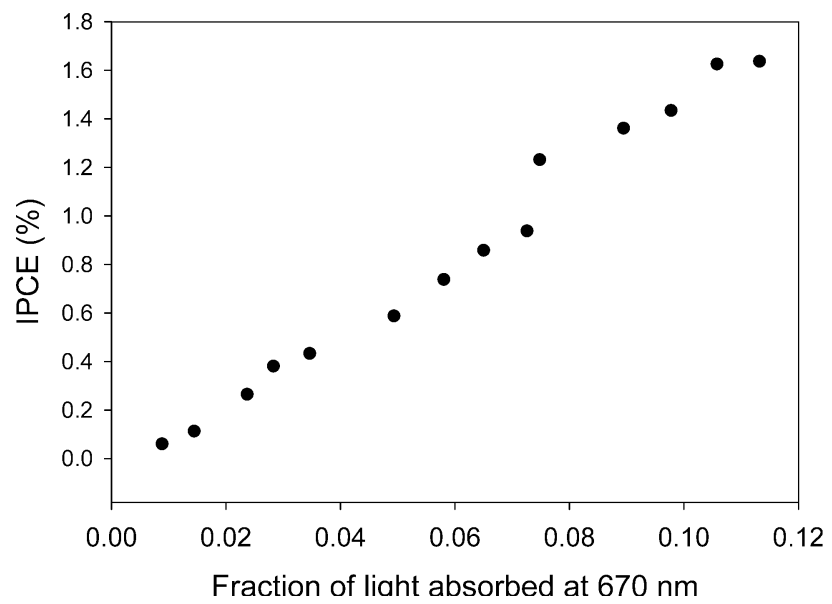

Figure 4. Incident photon-to-current efficiency (IPCE) as a function of the fraction of light absorbed by the photoelectrode at $670 \mathrm{~nm}$. (Larger IPCE values are observed at $440 \mathrm{~nm}$; cf. Figure 3.)

\section{Discussion}

The cathodic direction of the cell photocurrent rules out an excited state electron injection mechanism like that in Figure 1. From measurements with compound $\mathbf{2}$ in film environments, decay of the dye excited state is complex; reasonable fits are obtained with a biexponential function using lifetimes of $\sim 0.5$ and $\sim 1.7 \mathrm{~ns}$. Both components are sufficiently short that an

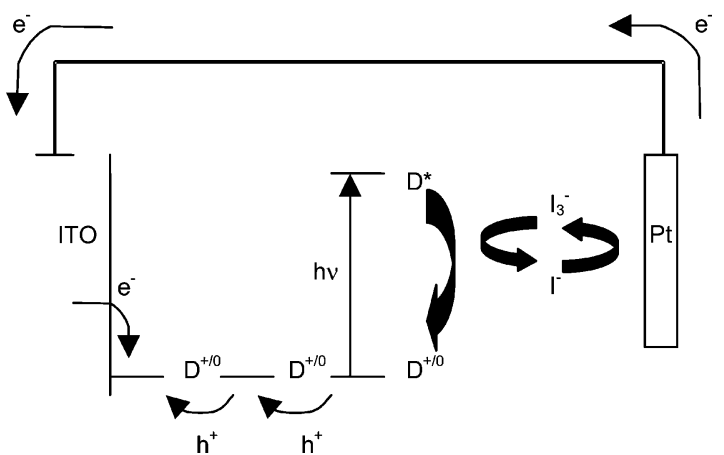

Figure 5. Mechanism of cathodic current generation by 2/ITO multilayer electrodes.

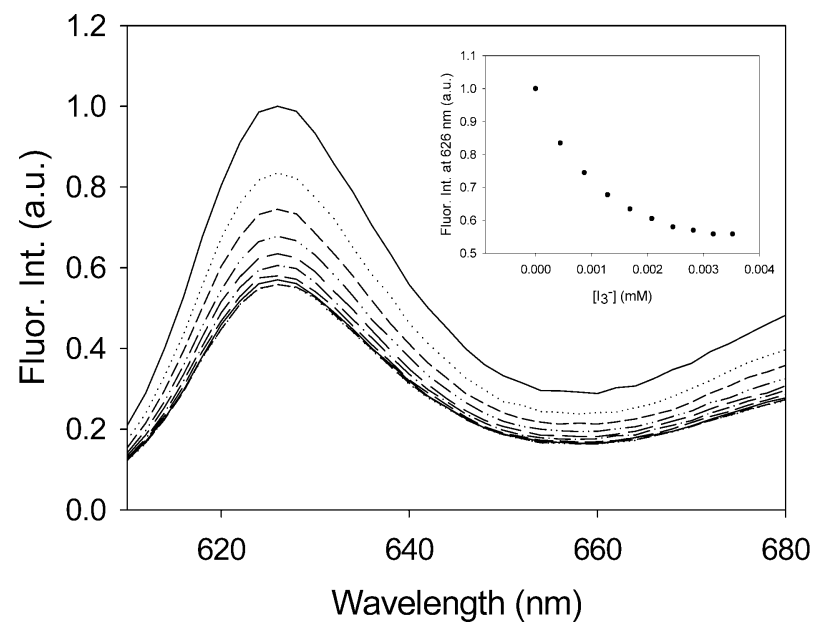

Figure 6. Fluorescence titration of $\mathbf{1}$ in $\mathrm{H}_{2} \mathrm{O}$ with $\mathrm{I}_{3}{ }^{-}$. Inset: Fluorescence intensity at $626 \mathrm{~nm}$ as a function of $\left[\mathrm{I}_{3}^{-}\right]$.

alternative mechanism involving lengthy exciton hopping and eventual hole injection from the excited state into ITO, followed by $\mathrm{I}_{3}{ }^{-}$regeneration of the light absorber from its reduced form, can likely be excluded. More reasonable is oxidative quenching of the excited state by $\mathrm{I}_{3}{ }^{-}$. The balance of the proposed mechanism is shown in Figure 5 where (a) the captured electron is transported by $\mathrm{I}^{-}$through the solution to the dark electrode, (b) the dye-localized hole hops from layer to layer until it reaches the dye/ITO interface, and (c) the electron from the dark electrode moves through the external circuit, reaching the photoelectrode, and reacting at the ITO/dye interface to regenerate the light absorbing, neutral form of the dye.

Clearly, in view of the short dye excited state lifetime, quenching cannot involve diffusional encounters with $\mathrm{I}_{3}{ }^{-}$. Instead, it must be occurring via a preformed complex:

$$
\begin{gathered}
\mathbf{2}+\mathrm{I}_{3}^{-} \Leftrightarrow\left[2: \mathrm{I}_{3}{ }^{-}\right] \\
{\left[2: \mathrm{I}_{3}{ }^{-}\right]+h v \rightarrow \mathbf{2}^{+}+3 \mathrm{I}^{-}}
\end{gathered}
$$

The equilibrium constant, $K$, for complex formation can be estimated from the $\mathrm{I}_{3}{ }^{-}$concentration dependence of the photocurrent. Using data from Figure $2, K$ is $40 \mathrm{M}^{-1}$. Figure 6 shows the outcome of a related experiment: fluorescence titration of $\mathbf{1}$ with $\mathrm{I}_{3}{ }^{-}$in water as solvent. ( $\mathbf{2}$ is insoluble in water and too weakly fluorescent in thin-film form to be easily studied.) As with the photocurrent measurements, the observed dye quenching occurs at $\mathrm{I}_{3}{ }^{-}$concentrations too low for diffusional quenching. Static quenching is indicated, with $K$ equaling $900 \mathrm{M}^{-1}$, a value not inconsistent with the photocurrent result given the differences in both chromophore and environment. A 
possible alternative explanation of the behavior in Figure 6 (but not Figures 2-4) is heavy-atom (iodine) enhanced singlettriplet intersystem crossing. Transient absorbance measurements showed, however, that the triplet yield is diminished rather than enhanced in the presence of $\mathrm{I}_{3}{ }^{-}$, leaving oxidative quenching of 1 by $\mathrm{I}_{3}{ }^{-}$as the most probable explanation..$^{22}$

Returning to Figure 4, the molecular quenching mechanism provides an explanation for the persistence of increased photocurrent production with increasing number of dye layers, even with as many as 15 layers. Molecular quenching can occur anywhere within the permeable film, not just at the film/ electrode interface, as would be the case for semiconductorbased quenching. Once quenched, the now oxidized dye can transfer its hole by ground state redox hopping. Because the hopping step occurs in the ground state, rather than in a shortlived excited state, hole transfer need not be rapid to generate increasing cathodic photocurrents-except to the extent that back-reaction between iodide and the oxidized dye occurs.

One consequence of the photoredox scheme in Figure 5 is small photovoltages. The potential of the dark electrode is governed by the $\mathrm{I}_{3}^{-} / \mathrm{I}^{-}$couple, whereas the photoelectrode's potential is a mixed potential defined by both $\mathrm{I}_{3}{ }^{-} / \mathrm{I}^{-}$and the square ${ }^{+/ 0}$ couple. If the $\mathrm{I}_{3}{ }^{-} / \mathrm{I}^{-}$couple is the dominant couple at the photoelectrode, then the overall cell is essentially a photodriven concentration cell and is capable, therefore, of generating only small potential differences. Experimentally, we find open circuit photovoltages of only about $200 \mathrm{mV}$.

Further consideration of the proposed scheme leads to the conclusion that it will become inoperable if $\mathrm{TiO}_{2}$ replaces ITO as the support for the dye because the electrons collected at the dark electrode will be insufficiently reducing to reach the $\mathrm{TiO}_{2}$ conduction band. To test this notion, the photoelectrode shown in Figure 7 was used. The left side of the electrode is dyecoated ITO; the right side is dye-coated $\mathrm{TiO}_{2}$. As shown in the bottom panel of the figure, spatially selective illumination produces a large cathodic photocurrent on the ITO side, but only a small anodic current on the titanium dioxide side. Presumably, the anodic current is generated via the conventional DSSC scheme of Figure 1.

Because only small photovoltages can be produced, the reverse photocurrent scheme is impractical for achieving significant energy conversion. Although photocurrent production via the scheme is precluded at $\mathrm{TiO}_{2}$, molecular quenching should still occur. To the extent that it does, photoexcited dyes will be unproductively consumed. Furthermore, the resulting oxidized dye may combine with $\mathrm{TiO}_{2}$-injected electrons from other dye molecules, causing loss of photocurrent. This may well account for the very low photocurrent observed for right-side illumination of the electrode shown in Figure 7.

Conceivably, similar parasitic behavior occurs with other types of DSSCs run in other environments. Iodide quenching of photoexcited $\mathrm{Ru}(\mathrm{bpy}) 3^{2+}$ and related dyes is known, ${ }^{23}$ as is the formation of an outer-sphere complex between both $\mathrm{I}^{-}$and $\mathrm{I}_{3}{ }^{-}$and a stable chromium(III) analogue of the oxidized form of "N3", ${ }^{24}$ the ruthenium dye used in the most efficient DSSCs. ${ }^{25}$ Finally, the observations here with porous dye layers on ITO are reminiscent of studies by Sereno and co-workers on photolysis of carotenoid dye multilayers on ITO. ${ }^{26,27}$ The system comprises one of the very few where multilayer sensitization occurs. As seen here with porous squares as the chromophoric element, current in the carotenoid cell flows in the direction opposite from that for a conventional DSSC. It seems likely that a similar molecular quenching mechanism is responsible.
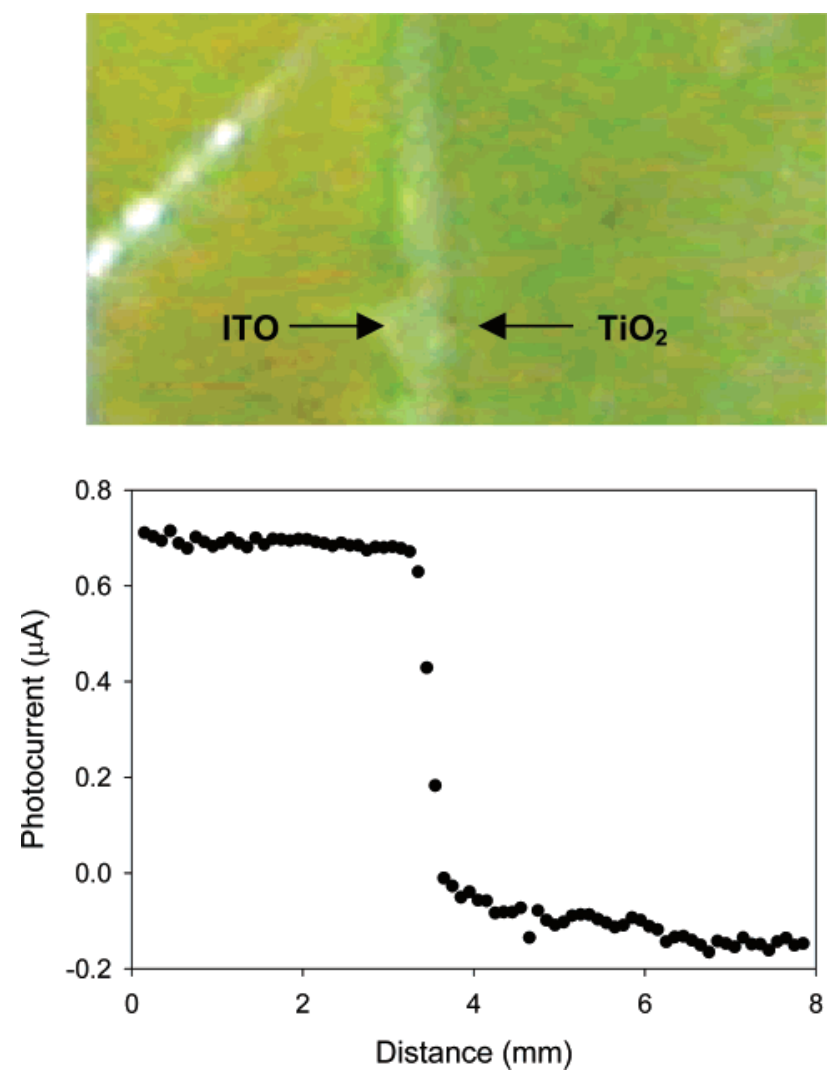

Figure 7. Top: ITO electrode containing areas of bare ITO and $\mathrm{TiO}_{2}$, and functionalized with square multilayers. Bottom: Photocurrent response as a function of illumination position on the slide. As the slide is translated the illuminated surface changes from $2 / \mathrm{ITO}$ to $2 / \mathrm{TiO}_{2} /$ ITO.

\section{Conclusions}

Photoelectrochemical cells based on porous porphyrinic assemblies as sensitizers display multilayer sensitization of ITO surfaces. The mechanism of sensitization is not excited state injection into the ITO electrode, but instead, $\mathrm{I}_{3}{ }^{-}$quenching of the porphyrin square excited state to produce an oxidized porphyrin. Quenching is possible, despite the short excited state lifetime, because of ground state donor (chromophore)/acceptor (quencher) complex formation. One consequence of the mechanism is that photocurrent flows in the direction opposite to that for conventional DSSCs. Another is that only small photovoltages can be produced. A third is that the photocurrent production scheme is inoperable when ITO is replaced by titanium dioxide, because the electrons produced are energetically incapable of reaching the $\mathrm{TiO}_{2}$ conduction band. Perhaps most importantly, to the extent that the first part of the scheme (molecular quenching) occurs in conventional cells, the photocurrent efficiencies of these cells will be diminished.

Acknowledgment. We thank Richard Gurney for providing samples of $\mathbf{2}$ and Emily Weiss for assistance with transient absorption measurements. We gratefully acknowledge financial support of the U. S. Department of Energy, Office of Science through grant no. DE-FG02-87ER13808. K.E.S. and A.M.M. additionally thank the Dow Foundation and Link Foundation, respectively, for graduate fellowship support.

\section{References and Notes}

(1) Hagfeldt, A.; Grätzel, M. Acc. Chem. Res. 2000, 33, 269-277.

(2) Hagfeldt, A.; Grätzel, M. Chem. Rev. 1995, 95, 49-68.

(3) Grätzel, M. Nature 2001, 414, 338-344. 
(4) Nazeeruddin, M. K.; Kay, A.; Rodicio, I.; Humphry-Baker, R.; Muller, E.; Liska, P.; Vlachopoulos, N.; Grätzel, M. J. Am. Chem. Soc. 1993, 115, 6382-6390

(5) Nazeeruddin, M. K.; Pechy, P.; Renouard, T.; Zakeeruddin, S. M.; Humphry-Baker, R.; Comte, P.; Liska, P.; Cevey, L.; Costa, E.; Shklover, V.; Spiccia, L.; Deacon, G. B.; Bignozzi, C. A.; Grätzel, M. J. Am. Chem. Soc. 2001, 123, 1613-1624.

(6) Alebbi, M.; Bignozzi, C. A.; Heimer, T. A.; Hasselmann, G. M.; Meyer, G. J. J. Phys. Chem. B 1998, 102, 7577.

(7) Fillinger, A.; Parkinson, B. A. J. Electrochem. Soc. 1999, 146, 4559

(8) Monat, J. E.; Rodriguez, J. H.; McCusker, J. K. J. Phys. Chem. A 2002, 106, 7399-7406.

(9) O'Regan, B.; Grätzel, M. Nature 1991, 353, 737-739.

(10) Nusbaumer, H.; Moser, J. E.; Zakeeruddin, S. M.; Nazeeruddin, M. K.; Grätzel, M. J. Phys. Chem. B 2001, 105, 10461-10464.

(11) Oskam, G.; Bergeron, B. V.; Meyer, G. J.; Searson, P. C. J. Phys. Chem. B 2001, 105, 6867-6873.

(12) de Jongh, P. E.; Vanmaekelbergh, D. Phys. Rev. Lett. 1996, 77, 3427-3430.

(13) de Jongh, P. E.; Vanmaekelbergh, D. J. Phys. Chem. B 1997, 101, $2716-2722$.

(14) Grätzel, M. J. Am. Ceram. Soc. 1997, 80, 3157-3171

(15) Moss, J. A.; Stipkala, J. M.; Yang, J. C.; Bignozzi, C. A.; Meyer, G. J.; Meyer, T. J.; Wen, X.; Linton, R. W. Chem. Mater. 1998, 10, 17481750 .

(16) Taniguchi, T.; Fukasawa, Y.; Miyashita, T. J. Phys. Chem. B 1999, 103, 1920-1924.
(17) Massari, A. M.; Gurney, R. W.; Huang, C.-H. K.; Wightman, M D.; Lozano, J.; Nguyen, S. T.; Hupp, J. T. Polyhedron 2003, 22, 30653072 .

(18) Katz, H. E.; Schilling, M. L.; Chidsey, C. E. D.; Putvinski, T. M; Hutton, R. S. Chem. Mater. 1991, 3, 699-703.

(19) Cao, G.; Hong, H.-G.; Mallouk, T. E. Acc. Chem. Res. 1992, 25, 420-427.

(20) Snover, J. L.; Byrd, H.; Suponeva, E. P.; Vicenzi, E.; Thompson, M. E. Chem. Mater. 1996, 8, 1490-1499.

(21) Legrand-Buscema, C.; Malibert, C.; Bach, S. Thin Solid Films 2002 $418,79-84$.

(22) Unfortunately, the transient absorbance experiments were ambiguous with respect to direct observation of the putative oxidized porphyrin.

(23) Nasr, C.; Hotchandani, S.; Kamat, P. V. J. Phys. Chem. B 1998, 102, 4944-4951.

(24) Walter, B. J.; Elliott, C. M. Inorg. Chem. 2001, 40, 5924-5927.

(25) In view of its extraordinarily high photocurrent efficiency and very rapid injection kinetics, the N3-based Grätzel cell itself is unlikely to suffer losses due to molecular quenching.

(26) Sereno, L.; Silber, J. J.; Otero, L.; del Valle Bohorquez, M.; Moore, A. L.; Moore, T. A.; Gust, D. J. Phys. Chem. 1996, 100, 814-821.

(27) Fungo, F.; Otero, L.; Durantini, E. N.; Silber, J. J.; Sereno, L.; Marino-Ochoa, E.; Moore, T. A.; Moore, A. L.; Gust, D. J. Phys. Chem. B 2001, 105, 4783-4790. 